\title{
Polymethylmethacrylate derivatives Eudragit E100 and L100 : Interactions and complexation with surfactants
}

\author{
Fabrice Ofridam ${ }^{1}$, Noureddine Lebaz ${ }^{1}$, Émilie Gagnière ${ }^{1}{ }^{\text {}}$, Denis Mangin ${ }^{1}$ and Abdelhamid \\ Elaissari $^{1}$ \\ ${ }^{1}$ Univ Lyon, University Claude Bernard Lyon 1, CNRS, LAGEPP UMR 5007, 43 boulevard du 11 \\ novembre 1918, F-69100, Villeurbanne, France
}

\begin{abstract}
:
Precipitation or coprecipitation of polyelectrolytes has been largely investigated. However, the precipitation of polyelectrolytes via addition of charged and non-charged surfactants has not been systematically studied and reported. Consequently, the aim of this work is to investigate the effect of different surfactants (anionic, cationic, non-charged and zwitterionic) on the precipitation of cationic and anionic polymethyl methacrylate polymers (Eudragit). The surfactants effect has been investigated as a function of their concentration. Special attention has been dedicated to the CMC range and to the colloidal characterization of the formed dispersions. Moreover, the effect of salt $(\mathrm{NaCl})$ and $\mathrm{pH}$ was also addressed. It is pointed out that non-ionic and zwitterionic surfactants do not interact with charged Eudragit E100 and L100. For oppositely charged Eudragit E100/SDS and Eudragit L100/CTAB, precipitation occurs, and the obtained dispersions have been characterized in terms of particle size distribution and zeta potential. It was established that the binding of SDS molecules to Eudragit E100 polymer chains is made through the negative charges of the surfactant heads under the CMC value whereas binding of CTAB to Eudragit L100 chains is made at a CTAB concentration 5 times above its CMC. For Eudragit E100/SDS system, a more acidic medium induces aggregation. A same result was observed for the Eudragit L100/CTAB at a more basic pH. Moreover, it was observed that increasing salt concentration (higher than $100 \mathrm{mM}$ ) led to aggregation as generally observed for polycations/anionic surfactant systems.
\end{abstract}

Keywords : Polyelectrolyte, polyelectrolyte-surfactant complexes, precipitation, pH- sensitive polymers, Eudragit.

\section{Introduction}

Polymers are nowadays used in various fields and are formulated in different ways according to the intended application. In fact, polymers find application in automotive and transport [1], electricity and electronics [2], building and civil engineering [3], cosmetics and fashion industry [4, 5], packaging and food engineering [6 8], medicine and health [9, 10] etc. For the latter application domain, the preparation of polymeric matrices as therapeutic vectors in the pharmaceutical industry is mostly based on precipitation methods using organic solvents [11. Polymers can also be precipitated from aqueous medium in uncharged state with the risk of collapse associated to the structure of the polymer chains 12,13 .

Precipitation or coprecipitation of polyelectrolytes has been largely investigated. This is the case for a well-known class of polymers referred to as smart polymers which are sensitive to external stimuli such as $\mathrm{pH}$, ionic strength, temperature, light, mechanical stress, electric and magnetic fields and ultrasounds [14. These polymers react to surrounding environmental conditions

*. Correspondence : Emilie Gagnière LAGEPP Université Claude Bernard Lyon 1, bât 308G ESCPE-Lyon, 43 bd du 11 Novembre 1918, Villeurbanne 69622 France, Email : emilie.gagniere@univ-lyon1.fr 
and can be soluble/insoluble depending on the abovementioned stimuli and their intensity [15]. Precipitation/solubilization property of $\mathrm{pH}$-sensitive polymers for instance finds application in drug delivery [16]. Another way of using polymers in drug delivery is through polyelectrolytes complexes (PECs). PECs are prepared by mixing cationic and anionic polymers in aqueous solutions with strong and reversible electrostatic interactions, conferring to the new elaborated coating material some specific properties [17]. Moreover, advances in drug delivery systems and polymer science have led to the development of another type of complexes known as polymer-surfactant complexes. In fact, polyelectrolyte-surfactant complexes (PESCs) find application not only in pharmaceutical formulations [18] but also in detergency [19], cosmetics [20], wastewater purification [21 23], rheological control [24,25] and in surface adsorption, biofuel extraction and oil recovery [26] and surface adsorption [27]. It was evidenced that surfactants performance could be bolstered with polymers addition. PESCs formation is known to be driven by electrostatic interactions between polymer chains and surfactant head groups that result in hydrophobic complexes [28,29. Mixtures of oppositely charged polymers and surfactants are prone to induce this kind of complexes. The process usually occurs at a surfactant concentration called the critical aggregation concentration (CAC), which is several orders of magnitude below the critical micelle concentration (CMC). Electrostatic interactions are influenced by the $\mathrm{pH}$, the surface charge density, and the salt concentration whereas hydrophobic interactions are sensitive to chain length, molecular weight or structure of the polyelectrolyte chains or surfactants tail. PESCs properties depend on surfactant concentration and mixing conditions as well. Altering these parameters induces macroscopic changes in the resulting nanosystems [30].

Poly (diallylammonium chloride) (PDADMAC) mixtures with different surfactants have been largely studied. This is due to the use of these mixtures in shampoo and hair conditioner formulations [31, 32]. Studies on polyacrylic acid (PAA) and poly methacrylic acid (PMA) interactions with surfactants have also been reported in literature [12, 13, 28, 33 36]. PESCs involving oppositely charged PAA and DoTab [37] or $\mathrm{C}_{14} \mathrm{TAB}[28]$ studies showed that their formation is related to the enhancement of hydrophobicity upon surfactant binding onto polymer chains. Furthermore, these studies reported the tendency of complex-complex interaction enhancement leading to the formation of aggregates and their gradual dissolution by increasing PAA molecular weight (MW) or in presence of surfactant excess. Deh-Ying Chu and J. K. Thomas established that there is a significant effect of the surfactant chain length and polyelectrolyte concentration on the CAC based on interactions between PMA and alkyl trimethylammonium bromide $\mathrm{C}_{n}$ TAB cationic surfactants 33. Moreover, Y. Li et al. investigated the interactions between Sodium Dodecyl Sulfate (SDS) and polyethylenimine (PEI) and some ethoxylated PEI [38]. They have firstly observed that for this system, the $\mathrm{CAC}$ of SDS decreases with decreasing $\mathrm{pH}$. They have further seen that SDS concentration range over which phase separation occurs decreases as the size of the ethoxylated chains increases. For the polymer with the longest ethoxylated chain, no precipitation occurs, and the number of bound surfactant molecules per mole of polymer is independent of $\mathrm{pH}$.

We previously investigated the effect of physico-chemical parameters such as $\mathrm{pH}$, salinity, polymer concentration, and incubation time on the solubility and the precipitation of two oppositely charged polymethylmethacrylate derivatives namely Eudragit L100 and Eudragit E100 [15]. Moreover, we studied the influence of a secondary polymer on the direct precipitation of these two polymers 17]. Eudragit L100 chains when solubilized present a negative charge due to the dissociation of carboxylic groups into $\mathrm{COO}^{-}$. Regarding Eudragit E100, its chains exhibit a positive charge due to the protonation of amine groups present in their structure $\left(\mathrm{NH}_{4}^{+}\right)$. Moreover, titration curves revealed two equivalences that helped estimating carboxylic content of Eudragit L100 (6 mmol $/ \mathrm{g})$ and ammonium content of Eudragit E100 (4 mmol/g). Starting from these preliminary results, the 
aim of the present work is to study charges screening effect of oppositely charged surfactants on the precipitation of the two Eudragit polymers. Formation and precipitation of PESCs, effect of pH and salt are studied and discussed throughout this work.

\section{Materials and methods}

\subsection{Materials}

Eudragit L100 (methacrylic acid - methyl methacrylate copolymer (1 :1)) powder (Mw= 125000 $\mathrm{g} / \mathrm{mol}$ ) and Eudragit E100 (dimethyl aminoethyl methacrylate, butyl methacrylate, and methyl methacrylate tri-copolymer with a ratio of $2: 1: 1$ ) pellets $(\mathrm{Mw}=47000 \mathrm{~g} / \mathrm{mol})$ were obtained from Evonik Röhm GmbH (Darmstadt, Germany). Sodium hydroxide was a product from Sigma Aldrich (Sweden) and hydrochloric acid (35\%) was purchased from VWR Chemicals (France). Sodium chloride was obtained from Laurylab (Brindas, France). Sodium dodecyl sulfate (SDS) purchased from Sigma Aldrich (USA), Hexadecyltrimethylammonium bromide (CTAB) from Alfa Aesar (Germany), polyethylene glycol tert-octyl phenyl ether (Triton X-100) from Fisher bioreagents (USA) and 3-( $N, N$-Dimethyl myristyl ammonio) propane sulfonate (SB3-14) from Sigma Aldrich (China) were used as surfactants. Figure 1 presents the chemical structures of the studied polymers. Table 1 summarizes some characteristics of the studied surfactants and Figure 2 gives their chemical structure.
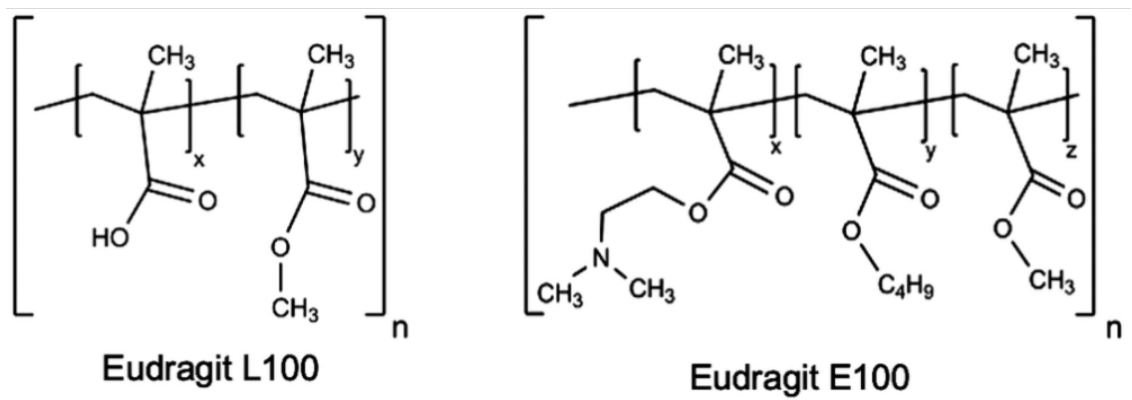

FiguRE 1 - Molecular structures of Eudragit L100 and Eudragit E100.

\begin{tabular}{|l|c|c|c|}
\hline Surfactant & Type & CMC (mM) & MW (g.mol $\left.{ }^{-1}\right)$ \\
\hline $\begin{array}{l}\text { Sodium Dodecyl Sulfate } \\
\text { (SDS) }\end{array}$ & Anionic & $7-10$ & 288.4 \\
\hline $\begin{array}{l}\text { Hexadecyl trimethyl ammo- } \\
\text { nium bromide (CTAB) }\end{array}$ & Cationic & 0.9 & 364.5 \\
\hline $\begin{array}{l}4-(1,1,3,3-T e t r a m e t h y l b u t y l) \\
\text { phenyl-polyethylene glycol } \\
\text { (Triton X-100) }\end{array}$ & Non-ionic & $0.17-0.3$ & 630 \\
\hline $\begin{array}{l}\text { 3-(N,N Dimethyl myristyl } \\
\text { ammonio) propane sulfonate } \\
\text { (SB3-14) }\end{array}$ & Zwitterionic & $0.1-0.4$ & 363.6 \\
\hline
\end{tabular}

TABLE 1 - Some characteristics of the investigated surfactants [39]. 

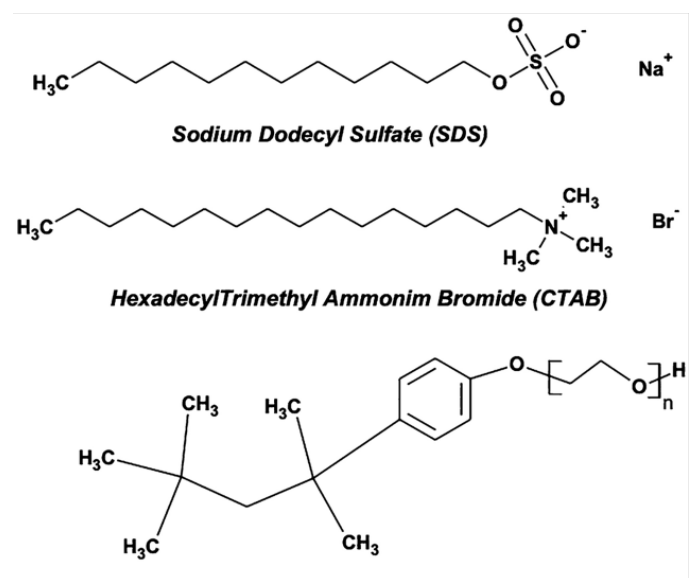

4-(1,1,3,3-Tetramethylbutyl)phenyl-polyethylene glycol (Triton X-100)

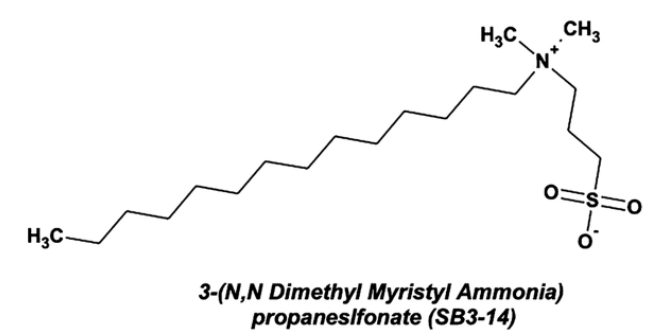

FiguRE 2 - Chemical structure of the investigated surfactants.

\subsection{Methods}

\subsubsection{Samples preparation}

All mixtures, solutions and samples were prepared and stored at room temperature. Eudragit L100 powder and Eudragit E100 pellets were firstly solubilized respectively in $\mathrm{NaOH}$ and $\mathrm{HCl}$ at a concentration of 2.5 g. $\mathrm{L}^{-1}$. Three final solubilization $\mathrm{pH}$ values were achieved for each polyelectrolyte (7, 13 and 14 for L100 and 2.5, 1, and 0 for E100). Surfactant solutions were prepared by solubilization in a medium prepared at the solubilization $\mathrm{pH}$ of the corresponding Eudragit polymer. Dilutions are then prepared to obtain the desired surfactant concentrations without $\mathrm{pH}$ variation. For low solubility surfactants such as CTAB, a slight heating was needed, and the experiments were realized quickly before any crystallization. If needed, a further slight heating was achieved before solution mixing.

Surfactant solutions were then mixed with polymer solutions in equivalent volumes in a one-shot procedure under continuous stirring at a rate of $500 \mathrm{rpm}$. It was shown that "one shot" or "stop-flow" mixing procedure resulted in PESCs particles that were much smaller in size and stable enough to be measured contrary to the dropwise or "slowly added" mixing method [40]. Moreover, it was shown that the smaller particles formed by the "surfactant to polymer" mixing method were more resistant to electrolytes induced colloidal instability than the larger ones formed by "polymer to surfactant" addition [41]. Different concentrations were tested for each surfactant by screening around its CMC whereas the polyelectrolyte concentration was kept constant at $0.125 \mathrm{wt} . \%$ in final samples. The $\mathrm{pH}$ of all surfactant solutions was adjusted to that of the corresponding polymer solution so that any further precipitation could not be linked to $\mathrm{pH}$ conditions. In a typical experiment, $40 \mathrm{~mL}$ of surfactant solution were added to $40 \mathrm{~mL}$ of polymer solution. Precipitation and polymer-surfactant 
complex formation was evaluated by macroscopic observation through turbidity appearance. The resulting suspensions were maintained under stirring for 30 minutes and were further characterized in terms of size distribution and zeta potential.

\subsubsection{Samples characterization}

Particle Size Distribution (PSD) Hydrodynamic mean particle size and size distribution of the precipitated polymers were determined by Dynamic Light Scattering (DLS) using a Zetasizer Nano ZS (Malvern Instruments) in the case of submicron particles and by light diffraction using a Mastersizer 3000 (Malvern Instruments) in the case of micrometric sized particles. Measurements were performed in deionized water for light diffraction and in $10^{-3} \mathrm{M}$ sodium chloride deionized water solution for DLS.

Zeta potential measurement Zeta potential (ZP) was deduced from electrophoretic mobility measurement of all obtained suspensions using a Zetasizer Nano ZS (Malvern Instruments, France). Measurements were performed at room temperature, $\mathrm{pH}$ of the prepared samples, in $10^{-3} \mathrm{M} \mathrm{NaCl}$ medium and under effective voltage of $149 \mathrm{~V}$ automatically set by the device. Each value is the average of more than 5 runs.

\section{Results and discussion}

\subsection{Non-ionic surfactant : Triton X-100}

The non-ionic surfactant effect on the cationic and anionic polyelectrolytes was investigated as a function of surfactant concentration. Surprisingly, no marked effect was observed irrespective of the polymer charge nature. For Eudragit E100 and L100, the investigated Triton X-100 concentration ranging from $0.1 \mathrm{mM}$ to $5 \mathrm{mM}$ (17-30 times the CMC value) revealed no precipitation of both polymers for the considered $\mathrm{pH}$ range ( $\mathrm{pH}=7$ to 14 for $\mathrm{L} 100$ and $\mathrm{pH}=0$ to 2.5 for E100). This can be attributed to a total lack of interaction between the polyelectrolytes and Triton X-100. In fact, Triton is a non-charged surfactant and both polyelectrolytes are soluble in the investigated $\mathrm{pH}$ ranges. This can also be attributed to static microstructure of the used polymers (E100 \& L100). The same results were obtained by E. Fegyver and R. Mészàros [42 regarding the interactions taking place between PDADMAC and nonionic surfactants $n$-dodecyl- $\beta$-D-maltoside $\left(\mathrm{C}_{12} \mathrm{G}_{2}\right)$ and $n$-dodecylhexa-ethylene-glycol $\left(\mathrm{C}_{12} \mathrm{E}_{6}\right)$. Moreover, Nagarajan analyzed the binding of poly (ethylene oxide) (PEO) to Triton $\mathrm{X}$ which presents a bulky neutral head [43]. He found that complexation was not favored due to free micelles formation instead of polymer-surfactant complexation. Based on this observation, the absence of interaction between Eudragit E100/L100 polyelectrolytes and Triton $\mathrm{X}-100$ could be attributed to the high water molecules-polymer interactions in the investigated $\mathrm{pH}$ domain irrespective of Triton X-100 concentration.

\subsection{Zwitterionic surfactant : SB3-14}

Phase behavior of polymers and zwitterionic surfactant mixtures has gained interest during the last decade. This is due to recent international regulation for a safe and healthy use of chemical compounds in consumer products. They recommended indeed a progressive replacement of charged molecules, either polymers or surfactants, by neutral or zwitterionic ones 44. In the present study, various SB3-14 zwitterionic surfactant (containing anionic carboxylate and cationic ammonium) concentrations were mixed with Eudragit E100 and L100 solutions. As for the non-ionic surfactant 
reported earlier, no effect has been observed irrespective of polymer nature, surfactant concentration ranging from 0.1 to $50 \mathrm{mM}$ (corresponding to 125-500 times the CMC value) and investigated $\mathrm{pH}$ domain ( $\mathrm{pH}=7$ to 14 for $\mathrm{L} 100$ and $\mathrm{pH}=0$ to 2.5 for E100). This result is quite surprising since this kind of surfactant exhibits both positive and negative charges making it suitable to interact basically with both polymers. The charge of the used polyelectrolyte is screened by the opposite charge of the zwitterionic surfactant. Instead of the polymer precipitation, the second residual charge of the surfactant promotes its solubilization. Consequently, as a result, the mixture solutions remain clear irrespective of surfactant concentration.

This observation is similar to the result obtained by E. Guzmán et al. [45] who noticed that there was no phase separation in mixtures of PDADMAC with sulfate-free zwitterionic surfactant coco betaine (CB) (i.e. no any observed opalescence). Their results confirmed by DLS and electrophoretic mobility measurements were explained by taking into account the zwitterionic aspect of the hydrophilic surfactant head. Indeed, the headgroup of this surfactant exhibits at the same time anionic carboxylate and cationic ammonium. Our observations are also in agreement with the results reported by O.Pyshkyna et al. [46]. By mixing poly( $N$-ethyl-4-vinylpyridinium bromide) and a zwitterionic surfactant ( $n$-Dodecyl-(3-sulfopropyl)ammonium), the resulting solutions remain transparent in a wide range of surfactant/polymer molar ratios (from 0.1 to 200). Further addition of sodium bromide produces charges screening leading to complexation and phase separation. They suggest the formation of a coacervate complex between the polycation and the zwitterionic surfactant at concentrations above its CMC.

Akanno et al. [47] suggested the presence of two different regimes for PDADMAC -zwitterionic surfactant binding. The first one concerns low surfactant concentrations where zwitterionic surfactant molecules bind to the polymer chains. At this stage, there is no significant charge neutralization (each neutralized charge is counteracted by the positive charge present in the surfactant molecule). The second regime is at high concentrations (molar ratio polymer/surfactant equal to 1) where surfactant binding occurs through micelles which enables a real neutralization of PDADMAC charges. Thus, they showed the existence of polyelectrolyte-zwitterionic surfactant complexation for a very high surfactant concentration. They observed a significant micelle induced complexation for a mixture of PDADMAC and coco-amidopropyl-betaine only for a surfactant concentration 333 times (100 $\mathrm{mM}$ ) the CMC value. Evidence of polymer-surfactant complex formation was only found when the number of surfactant molecules was close to the number of charged monomers in solution. Surfactant micelle negative charges interact with polymer monomers. The same result tendency was observed in micelle formation regimen for a mixture of SB3-14 with polycationic Eudragit E100 for a surfactant concentration of $100 \mathrm{mM}$ (250-1000 times the CMC value). At the same SB3-14 concentration, no precipitation occurred while working with polyanionic Eudragit L100.

\subsection{Anionic surfactant : SDS}

The effect of SDS on precipitation of both Eudragit E100 and L100 was investigated in the range of $1 \mathrm{mM}$ to $20 \mathrm{mM}$ (2-3 times its CMC value). All mixtures performed using Eudragit L100 exhibit a clear aspect which is explained by a total absence of interactions between both negatively charged SDS and Eudragit L100. This can be attributed to non-attractive interaction. As expected, due to negative charge of both Eudragit L100 and SDS, attractive electrostatic interactions are excluded. This also shows absence of hydrophobic interactions.

However, the effect of SDS on Eudragit E100 was found to be more relevant. From experiments 
performed at $\mathrm{pH}=2.5$, three SDS concentration domains are pointed out. For low concentrations $(<$ $4 \mathrm{mM}$ ), the amount of SDS is not sufficient to induce any screening effect. When the SDS concentration reaches $4 \mathrm{mM}$, milky like dispersions were obtained revealing the precipitation of Eudragit E100 (Figure 3). This is due to the sufficient SDS amount for the screening of Eudragit E100 charges and consequently reducing the solubility of the polyelectrolyte which induces polymer precipitation. Eudragit E100 positive charges are screened by SDS negative charges leading to precipitation following the scheme proposed in Figure 4.

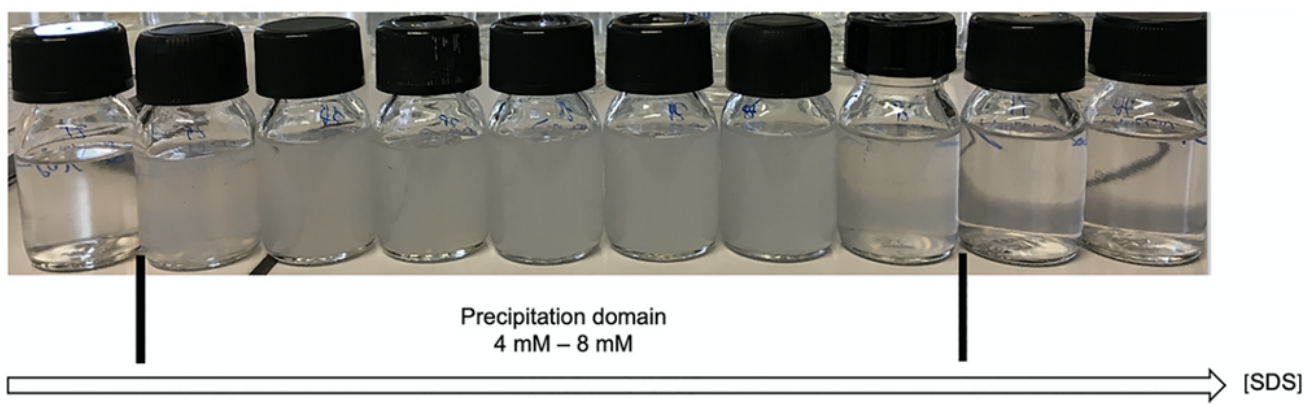

FIgURE 3 - Picture showing the three SDS interaction domains with Eudragit E100. Left : SDS concentration $<4 \mathrm{mM}$, no screening effect, no precipitation. Middle : complexation and Eudragit E100/SDS precipitation (4-8 mM). Right : Complexes solubilization upon micelles formation beyond $8 \mathrm{mM}$.
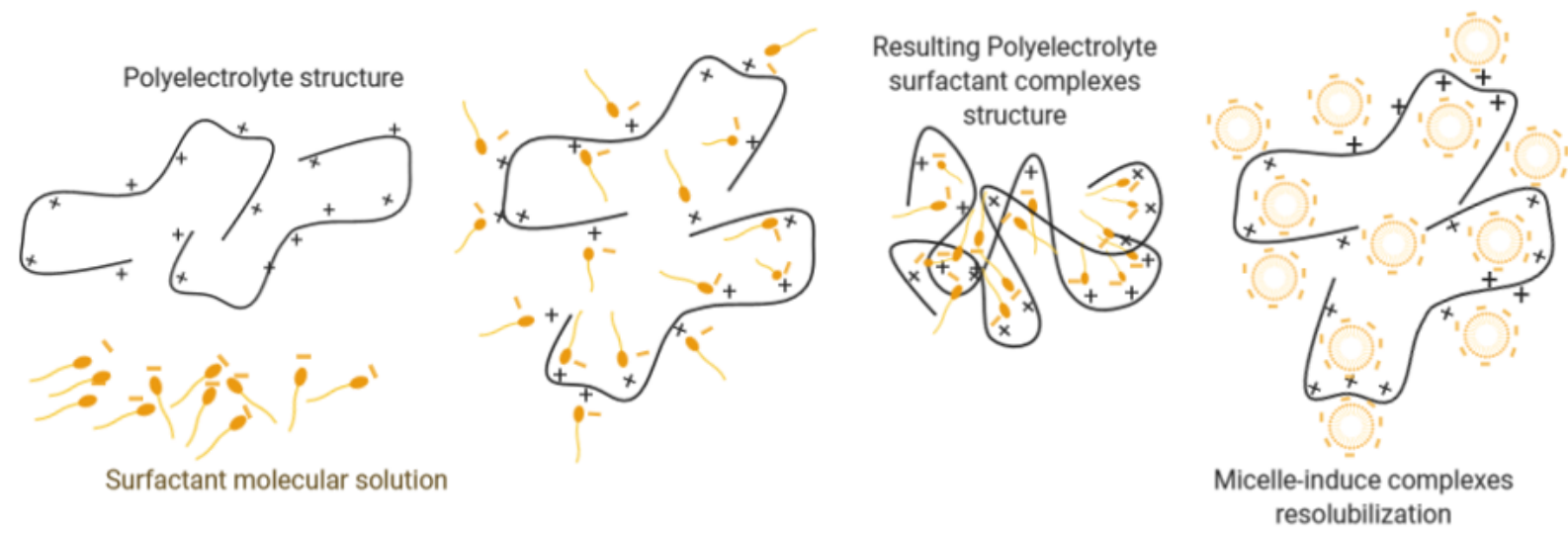

FIGURE 4 - A scheme illustrating interactions between positively charged Eudragit E100 chains and negatively charged SDS molecules headgroups as a function of SDS concentration. (a) SDS molecules binding to Eudragit E100 chains. (b) Polyelectrolyte chains collapse as Eudragit E100-SDS complex. (c) Complex resolubilization occurred in micellar regimen.

Normally, for this surfactant/polymer charge ratio (0.4), the zeta potential should correspond to the isoelectric point. Above this SDS concentration, the formed SDS micelles interact with the positive charges of the polymer. Then, instead of charge screening, the immobilized micelles on the polymer via attractive electrostatic interactions enhance the solubilization of the SDS-polymer complexes. 
The obtained dispersions (milky like dispersions) in the precipitation domain discussed above are globally submicronic in size as illustrated in Figure 5 in which size distributions deduced from light scattering and diffraction are presented. Precipitation occurred for a polymer to surfactant charge ratio ranging from 1.25 to 2.5. The obtained hydrodynamic sizes from light scattering show both narrow size distribution and bimodal for $4 \mathrm{mM}$ SDS concentration. But the average hydrodynamic size remains submicronic in nature. The optimum SDS concentration induces large particles size as determined via diffraction analysis, shows narrow size distribution ranging from around $100 \mathrm{~nm}$ to $1000 \mathrm{~nm}$, but remains submicronic too (Figure 5).
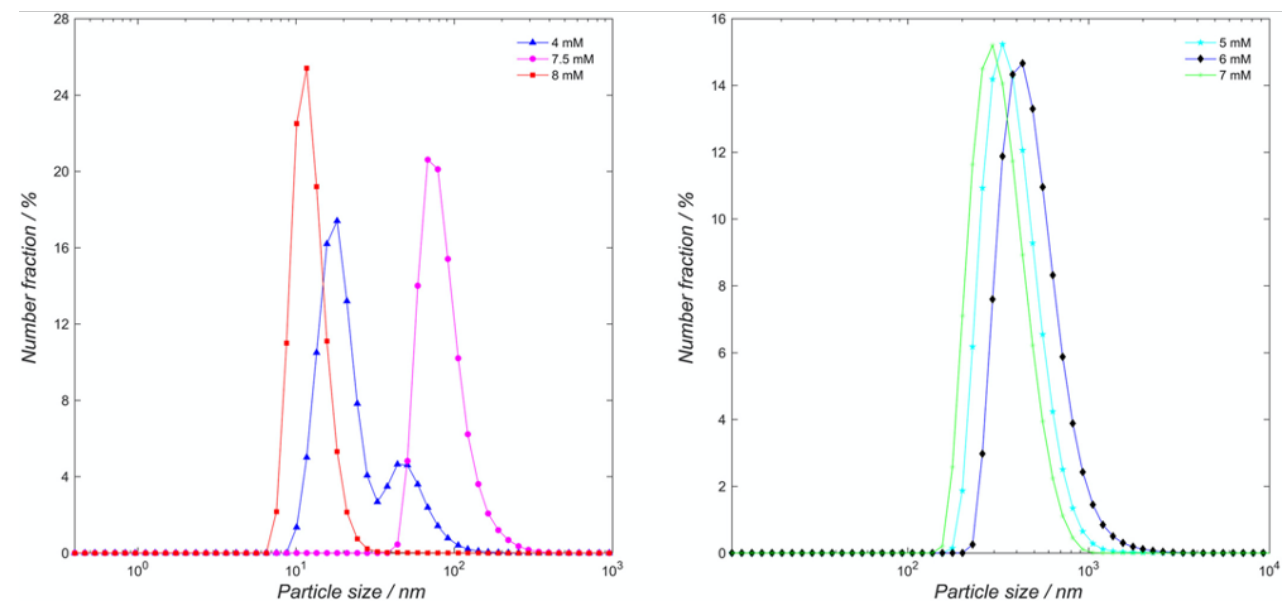

FigURE 5 - Number-based particle size distribution of E100/SDS particles as a function of SDS concentration : DLS (left) and light diffraction (right) measurements.

It can be observed that elaborated complexes mean size increases when increasing surfactant concentration from 4 to $6 \mathrm{mM}$. At this point, a maximum mean size seems to be reached. A. Akanno et al. 48 pointed out the fact that turbidity increase in the ascendant part of the precipitation domain is most likely linked to the increase in aggregates size. The formation of complexes involves several polymer chains. Thus, the increasing turbidity is not linked to the formation of more chargecompensated complexes in the bulk phase. Turbid dispersions obtained in our experiments associated with non-neutral ZP measurements support otherwise the hypothesis that the turbidity increase is related to kinetically formed aggregates during the preparation process. Working on phase behavior of PDADMAC and sodium $N$-lauroyl- $N$-methyltaurate aggregates, S. Llamas et al. [49] reported the same tendency. They observed an increase in turbidity and apparent hydrodynamic radius when increasing surfactant concentration in a precise range.

For SDS concentration between $7 \mathrm{mM}$ and $8 \mathrm{mM}$, a decrease of the mean size is observed. This means that for this range, an increase of SDS concentration produces the inverse effect. The resulting system exhibits a slightly bluish appearance at 7.5 and $8 \mathrm{mM}$. In other words, turbidity is low at low surfactant concentrations, increases and reaches a maximum before decreasing when the surfactant concentration is high. This is consistent with what was observed by other authors for polycationsanionic surfactant bulk phase behavior [41,50]. Inside the precipitation domain, minimal mean size is associated with the highest and the lowest concentrations. Between them, there is an increase of the mean size following turbidity trend as shown in Figure 6. 


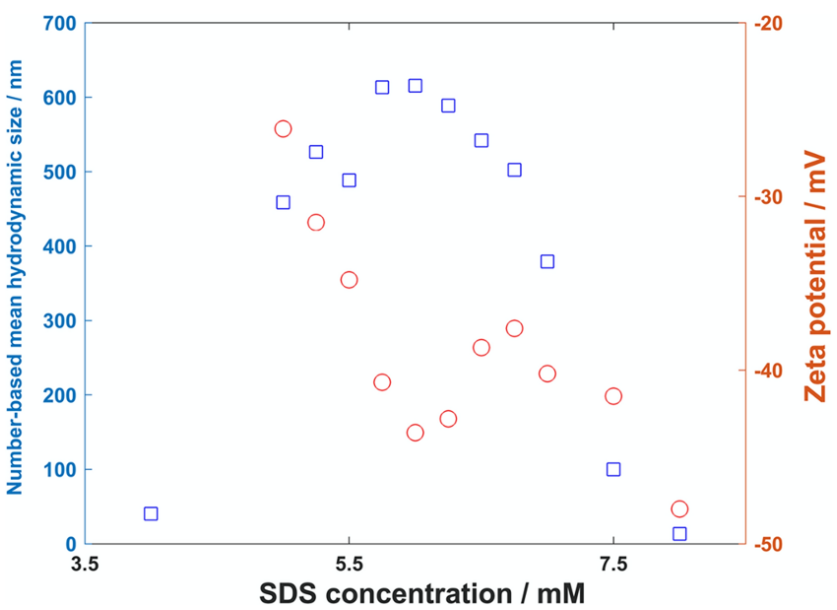

FiguRE 6 - Number-based mean hydrodynamic size (squares) and Zeta potential (circles) of Eudragit E100/SDS particles as a function of SDS concentration.

In addition to Eudragit E100/SDS mean particle size, Figure 6 presents the evolution of particles $\mathrm{ZP}$ as a function of SDS concentration. The ZP of the obtained dispersions was found to be negative. It reveals the possible restructuration of SDS molecules in the formed aggregates. The zeta potential measured in acidic conditions for the milky dispersions was high enough (in absolute value) to guarantee the aggregates stabilization for at least 24 hours. Sedimentation occurred with an extended storage time. Indeed, zeta potential value ranged from $-26 \mathrm{mV}$ at $5 \mathrm{mM}$ to $-40.2 \mathrm{mV}$ at $7 \mathrm{mM}$ and $\mathrm{pH}=3$ (Figure 6). $\mathrm{ZP}$ was not measured as a function of $\mathrm{pH}$ since Eudragit E100 is $\mathrm{pH}$-sensible which affects polymer solubilization and also conformation. Eudragit E100/SDS particles gradually take surfactant charges as its concentration is increased. This is in agreement with what was reported in the literature 30$]$.

The third domain of the phase behavior of Eudragit E100/SDS mixtures is defined for SDS concentration beyond $8 \mathrm{mM}$. For this concentration range, the system (Eudragit E100/SDS mixtures) was found to be totally non-turbid (see Figure 3). When increasing surfactant concentration from neutralization concentration point (CNC) to CMC and further, many authors have observed the nonformation of complexes. This can be attributed to micelles interactions with the oppositely charged polymer inducing good solubilization rather than precipitation via charges screening as discussed above.

\subsection{Cationic surfactant : CTAB}

The effect of CTAB on precipitation of both Eudragit E100 and L100 was investigated as a function of surfactant concentration ranging from $0.1 \mathrm{mM}$ to $20 \mathrm{mM}$ (20 times the CMC value). As expected, no effect of CTAB on cationic Eudragit E100 was observed. This is due to repulsive electrostatic interactions and also to the marginal effect of attractive hydrophobic interactions.

As for Eudragit E100/SDS system, three CTAB concentration domains can also be identified for Eudragit L100/CTAB system. For low concentrations (i.e. below $4.5 \mathrm{mM}$ ), the amount of CTAB is not sufficient to induce any screening effect of oppositely charged chains. By increasing CTAB concentration above $4.5 \mathrm{mM}$ (five times $\mathrm{CMC}$ value) at a $\mathrm{pH}=7$, bluish dispersions are obtained. This defined the second CTAB domain (see Figure 7). 


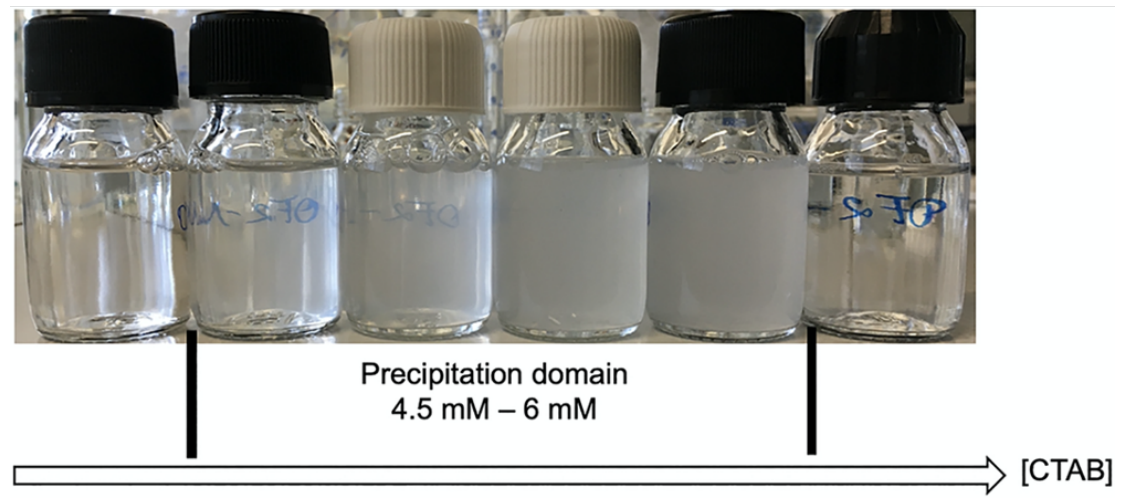

Figure 7 - Picture showing the three CTAB interactions domains with Eudragit L100. Left : CTAB concentration $<4.5 \mathrm{mM}$, no screening effect, no precipitation. Middle : complexation and Eudragit L100/CTAB precipitation (4.5-6 mM). Right : Complexes solubilization for high CTAB concentrations (beyond $6 \mathrm{mM}$ ).

In fact, by increasing surfactant concentration, increased turbidity is observed. For CTAB concentrations ranging from 5 to $6 \mathrm{mM}$, milky dispersions are obtained. Based on the explanations given previously, it can be deduced that the cationic charges of CTAB gradually screened Eudragit L100 negative charges. Contrary to Eudragit E100, solubilized Eudragit L100 presents negatively charged pendant groups along his chain 15. Positive charges brought by the surfactant counterbalance the polymer negative charges and induce phase separation. The phenomenon occurred for polymer to surfactant charge ratio of approximatively 2.5 to 3.5 following the micelle-induced precipitation mechanism that is illustrated in Figure 8.
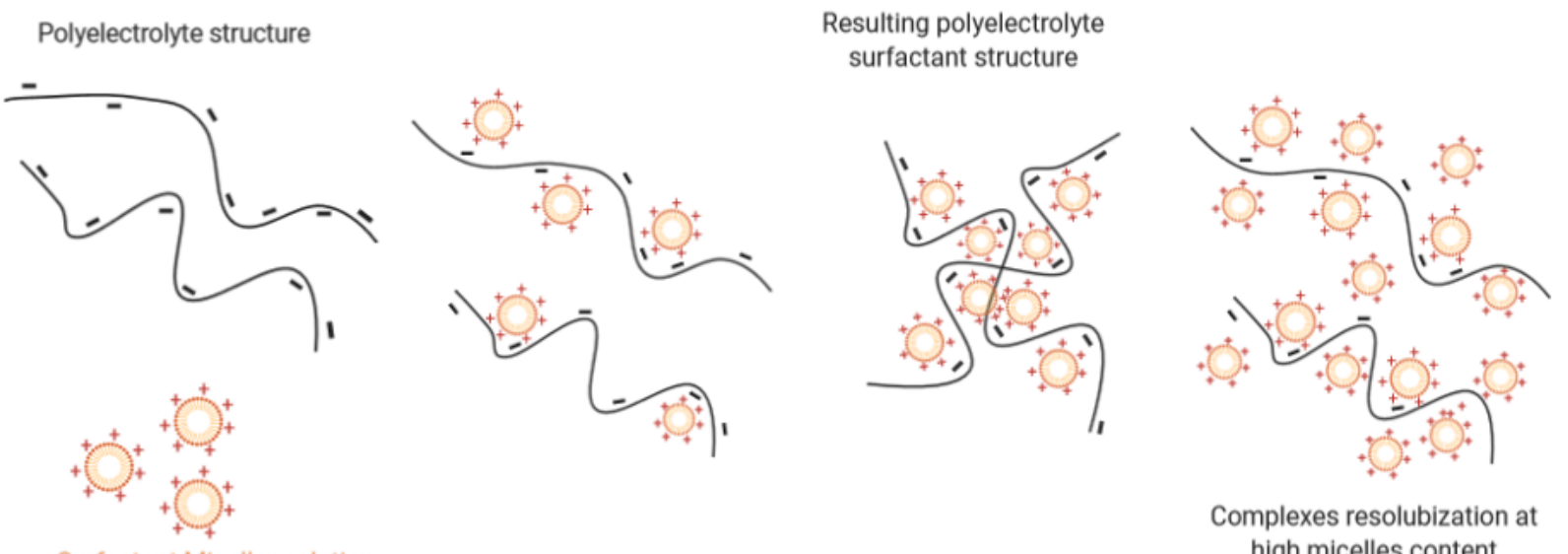

Complexes resolubization at high micelles content

FigURE 8 - A scheme illustrating interactions between negatively charged Eudragit L100 polyelectrolyte chains and positively charged CTAB micelles. (a) CTAB micelles binding to Eudragit L100 chains. (b) Polyelectrolyte chains collapse as Eudragit L100-CTAB complex. (c) Complex resolubilization occurred at high micelles content. 
For this precipitation domain, Figure 9 presents size distributions with their corresponding mean diameter and zeta potential of Eudragit L100/CTAB particles as a function of CTAB concentration. Contrary to what was observed for the previous Eudragit E100/SDS system, there is no marked effect of surfactant concentration inside the precipitation range on particles size distribution.
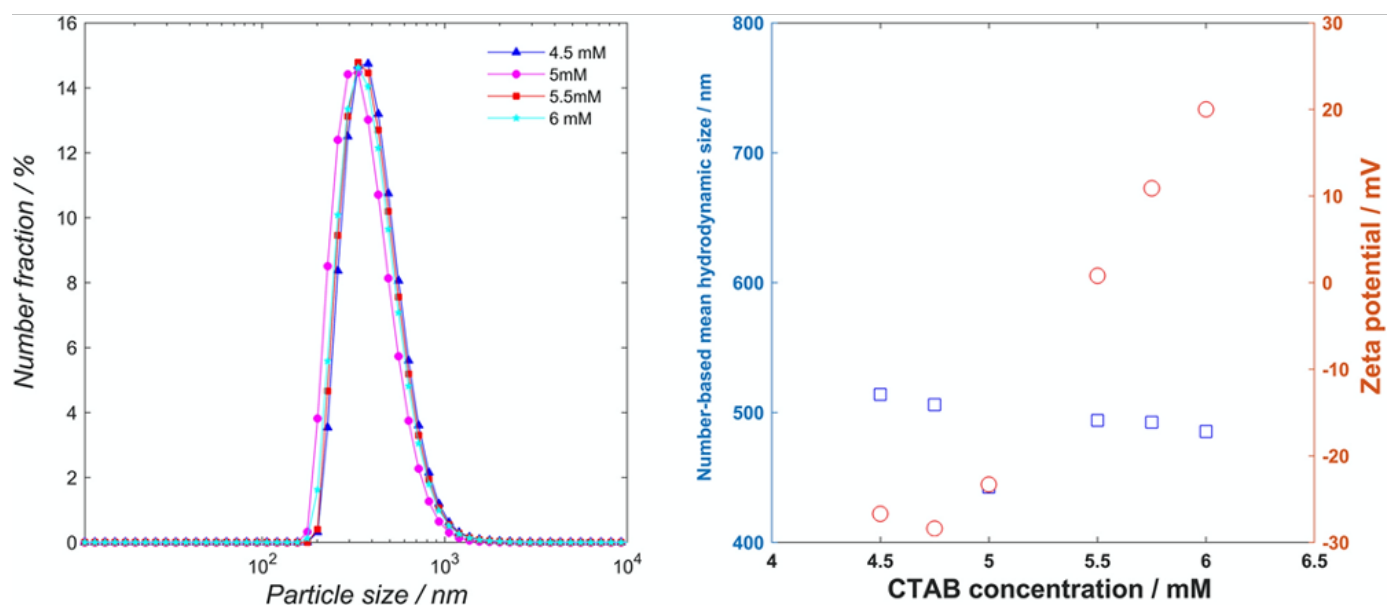

FigurE 9 - Number-based size distribution of L100/CTAB particles as a function of CTAB concentration (left) Number-based mean size (squares) and Zeta potential (circles) of Eudragit L100/CTAB particles as a function of CTAB concentration (right).

Zeta potential increased with increasing CTAB concentration from $-26.7 \mathrm{mV}$ at $4.5 \mathrm{mM}$ to +20 $\mathrm{mV}$ at $6 \mathrm{mM}$. This increase of $\mathrm{ZP}$ is in agreement with what was stated before for Eudragit E100/SDS system.

The third domain is defined for CTAB concentration beyond $6 \mathrm{mM}(\simeq 7$ times $\mathrm{CMC}$ value). For this concentration range, the concentration of micelles is sufficient not only to interact with the oppositely charged domains on the polymer chain, but also to enhance the solubility of the forming complexes. In fact, the charges screened by adsorbed CTAB polar parts are replaced by non-involved molecules, leading consequently to more water complexes [40] (see Figure 7).

\subsection{Effect of $\mathrm{pH}$ on the precipitation of oppositely charged Eudragit L100/CTAB and Eudragit E100/SDS}

The effect of $\mathrm{pH}$ on the bulk phase behavior of oppositely charged systems Eudragit E100/SDS and Eudragit L100/CTAB was also investigated. For this purpose, Eudragit E100 and L100 were solubilized respectively in more acidic and more basic mediums. The same experimental protocol as described before was followed. Surfactant concentration was set to $5 \mathrm{mM}$ and $4.5 \mathrm{mM}$ respectively for SDS and CTAB.

First, the $\mathrm{pH}$ (in the investigated range) has no drastic effect on the investigated polyelectrolytes and surfactants.

The effect of $\mathrm{pH}$ on E100/SDS system was performed for $\mathrm{pH}$ values ranging from 0 to 2.5 and the hydrodynamic particle size was found to increase with decreasing the $\mathrm{pH}$. This can be mainly attributed to salt effect rather than $\mathrm{pH}$ effect. In fact, too acidic $\mathrm{pH}$ induces high salinity which is 
known to affect colloidal stability by inducing aggregation phenomena and consequently large particle size. Another significant observation that is made from this series of experiments is the larger precipitation domain. Precipitation already occurs at SDS concentration of $3 \mathrm{mM}$. As for example, turbid samples were observed for SDS concentrations ranging from 3 to $15 \mathrm{mM}$ and 3 to $25 \mathrm{mM}$ respectively for experiments performed at $\mathrm{pH}=1$ and 0 .

On the other hand, the effect of $\mathrm{pH}$ on L100/CTAB was also investigated in the $\mathrm{pH}$ range from 7 to 14 . The polymer Eudragit L100 is negatively charged in this pH range and totally soluble. The ionic strength increases with increasing the $\mathrm{pH}$ and consequently, large particles/aggregates are formed. Such behavior is similar to E100/SDS at acidic pH values as discussed above. Here also, larger precipitation domains were observed. Precipitation occurred for CTAB concentration from 4 to $10 \mathrm{mM}$ for experiments performed at $\mathrm{pH}=13$ and 14 for instance.

\subsection{Effect of salt on the precipitation of oppositely charged Eudragit L100/CTAB and Eudragit E100/SDS}

Effect of $\mathrm{NaCl}$ on the bulk phase behavior of oppositely charged Eudragit E100/SDS and Eudragit L100/CTAB systems was also investigated. $\mathrm{NaCl}$ concentration ranging from 0 to $1000 \mathrm{mM}$ was studied by simple addition during the mixing process. The experiments were performed at $\mathrm{pH}$ values of 2.5 and 7 respectively for Eudragit E100/SDS and Eudragit L100/CTAB systems. Surfactants concentrations that were retained for salt concentration influence investigation were $5 \mathrm{mM}$ for SDS and $4.5 \mathrm{mM}$ for CTAB. As expected, salinity was found to have marked effect.

Regarding Eudragit E100/SDS, for low salinity below $50 \mathrm{mM}$, no marked effect was observed. In fact, milky like dispersions were obtained with comparable turbidity intensities and the hydrodynamic size was found to be almost the same as reported in Figure 10.
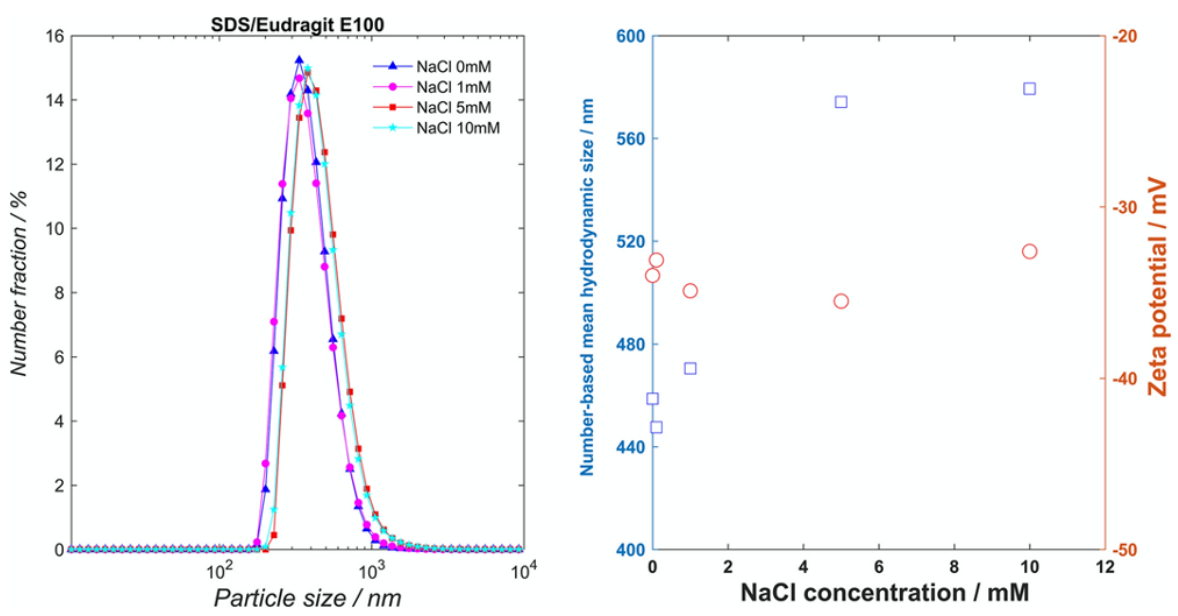

FiguRE 10 - Number-based particles size distribution of E100/SDS particles as a function of $\mathrm{NaCl}$ concentration, $[\mathrm{SDS}]=5 \mathrm{mM}, \mathrm{pH}=2.5$ (left). Number-based mean size (squares) and Zeta potential (circles) of Eudragit E100/SDS particles as a function of $\mathrm{NaCl}$ concentration $[\mathrm{SDS}]=5 \mathrm{mM}, \mathrm{pH}=2.5$ (right).

This can be attributed to negligible salinity effect on attractive electrostatic interactions and in another hand to high surface charge density (i.e. high zeta potential) of the formed particles, 
revealing good colloidal stability of the obtained dispersion. For high salinity (in the range of 100 to $1000 \mathrm{mM}$ ), large particles are obtained demonstrating that ionic strength induces aggregation phenomena of the primary formed particles. Naderi et al. [41] observed that high $\mathrm{NaCl}$ concentration induced instabilities in samples prepared by surfactant to polymer mixing method with sediment appearing even under stirring after 1000 hours. Wang et al. [51] also reported similar results for a polycation/SDS system as they observed that $\mathrm{NaCl}$ concentration above $0.1 \mathrm{M}$ is helpful to enhance complexes formation. The Figure 10 also shows the plot of zeta potential against $\mathrm{NaCl}$ concentration. The increase in $\mathrm{NaCl}$ concentration does not induce any marked effect on the zeta potential value. This is in agreement with the reported slight increase in particle mean size.

Regarding the Eudragit L100/CTAB system, three domains are observed as a function of $\mathrm{NaCl}$ concentration. First, for low ionic strength (0 to $10 \mathrm{mM}$ ), similar to E100/SDS, no effect was observed, and the average hydrodynamic size of obtained dispersions is almost in the same range (Figure 11).
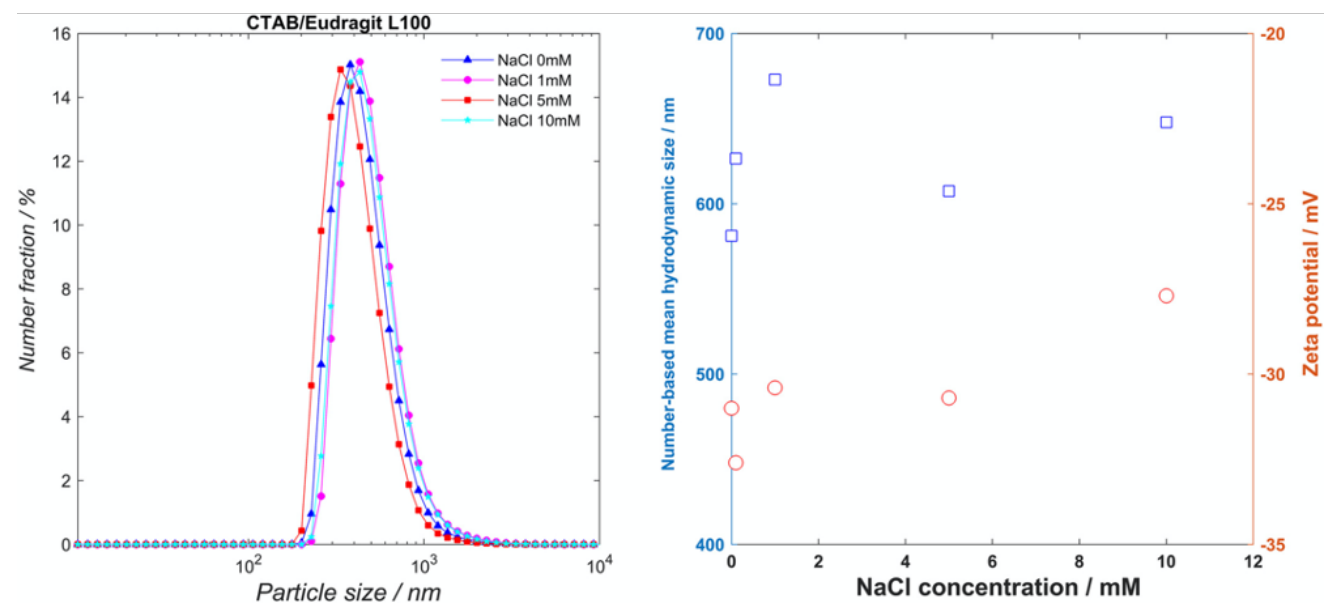

FiguRE 11 - Number-based particle size distribution of L100/CTAB particles as a function of NaCl concentration, $[\mathrm{CTAB}]=4.5 \mathrm{mM} ; \mathrm{pH}=7$ (left). Number-based mean hydrodynamic size (squares) and Zeta potential (circles) of Eudragit L100/CTAB as a function of $\mathrm{NaCl}$ concentration, $[\mathrm{CTAB}]=4.5$ $\mathrm{mM}, \mathrm{pH}=7$ (right).

However, aggregated dispersions were obtained around $50 \mathrm{mM}$ revealing the instability of the formed particles [41]. Surprisingly, for high salinity (i.e. above $50 \mathrm{mM}$ ), no formation of particles was observed. This can be attributed to (i) the screening effect of attractive electrostatic interactions between negatively charged polymer and positively charged surfactant and consequently no particles formation was observed and (ii) the possible reduction of CMC of CTAB surfactant leading consequently to a shift in the precipitation range. K. Pojjaźk et al. [40] reported for polyanionic polymer PSS/CTAB system that the application of high salt concentrations affects the equilibrium phase properties. They observed a considerable decrease of the amount of surfactant bound to the polyelectrolyte. The system tends to be converted as what we observed from a colloidal dispersion to a thermodynamically stable solution of polyelectrolyte and surfactant molecules. They linked this fact to repulsive hydration forces developed between the particles at high ionic strengths.

In brief, the effect of surfactant nature in the presence of Eudragit E100 and L100 polymers was investigated. Non-ionic surfactant Triton X-100 and zwitterionic one SB3-14 have shown no effect 
when added to polymers. Regarding oppositely charged polymer/surfactant systems, precipitation domains have been pointed out as function of surfactant concentration (Figures 4 and 8). Submicronic dispersions of Eudragit E100/SDS and Eudragit L100/CTAB were obtained. In the case of Eudragit E100/SDS, it was shown that particle size in the precipitation domain depends on SDS concentration. Regarding the effect of $\mathrm{pH}$ and salinity, it was observed that increasing salinity induced an increase of particle size. Surprisingly, for Eudragit L100/CTAB system, no phase separation was observed above $\mathrm{NaCl}$ concentration of $50 \mathrm{mM}$.

\section{Conclusion}

In this study, the effects of surfactant nature and concentration on Eudragit L100 and E100 precipitation were investigated. The results obtained for non-ionic and zwitterionic surfactants are in agreement with those already reported in literature. In fact, these two surfactants do not affect the solubility of both charged polyelectrolytes Eudragit L100 and E100. For non-ionic surfactants, this can be attributed to non-interactions with both polymers. Whereas, for the zwitterionic surfactant, the non-effect was attributed to the non-involved second charge on the surfactant which may enhance the solubilization compared to the involved first charge.

Regarding the effect of charged surfactants on similarly charged polyelectrolytes, no effect has been observed as expected. This has been attributed to the total absence of hydrophobic interactions between alkyl chains of the surfactants and the non-polar segments of the used polyelectrolytes. Whereas, the effect of charged surfactants on oppositely charged polyelectrolytes was found to be more marked. In fact, for both cases Eudragit L100/CTAB and E100/SDS, nanoprecipitation has been observed when the surfactant concentration is sufficient to induce polyelectrolyte charges screening. For high surfactant concentration (i.e. largely higher than the corresponding CMC), the polyelectrolyte/micelles interaction do not induce any precipitation. Despite the partial screening of the charges of the polyelectrolyte, the excess of the charges brought by the micelles induces the solubilization of the formed polymer/micelles complexes.

\section{Conflict of interest}

The authors declare no conflict of interest.

\section{Références}

[1] S.A. Begum, A.V. Rane, and K. Kanny. Chapter 20 - applications of compatibilized polymer blends in automobile industry. In A. A.r. and S. Thomas, editors, Compatibilization of Polymer Blends, page 563-593. Elsevier, 2020.

[2] J.G. Drobny. Polymers for electricity and electronics : materials, properties, and applications. John Wiley Sons, 2012.

[3] L.C. Hollaway. The evolution of and the way forward for advanced polymer composites in the civil infrastructure. Constr. Build. Mater, 17(6) :365-378, 2003.

[4] A. Lund, N.M. Velden, N.-K. Persson, M.M. Hamedi, and C. Müller. Electrically conducting fibres for e-textiles : An open playground for conjugated polymers and carbon nanomaterials. Mater. Sci. Eng. R Rep, $126: 1-29,2018$.

[5] G. Savary, M. Grisel, and C. Picard. Cosmetics and personal care products. In O. Olatunji, editor, Natural Polymers : Industry Techniques and Applications, page 219-261. Springer International Publishing, Cham, 2016.

[6] F. Froiio. Essential oils-loaded polymer particles : Preparation, characterization and antimicrobial property. Polymers, 11(6) :1017, 2019. 
[7] K.M. Hammi, M. Hammami, C. Rihouey, D.Le Cerf, R. Ksouri, and H. Majdoub. Ultrasonication of polysaccharides from tunisian zizyphus lotus fruit : Emulsifying capacities, rheological properties and antioxidant activities. Chem. Afr, 2020.

[8] S.K. Shukla, A.Bharadvaja Rizwana, and G.C. Dubey. Micro-cellulose sheet and polyvinyl alcohol blended film for active packaging. Chem. Afr, 2(4):723-732, 2019.

[9] A.Christy Hunter and S.Moein Moghimi. Smart polymers in drug delivery : a biological perspective. Polym. Chem, 8(1) :41-51, 2017.

[10] P.A. Gunatillake and R. Adhikari. 2 - nondegradable synthetic polymers for medical devices and implants. In L. Poole-Warren, P. Martens, and R. Green, editors, Biosynthetic Polymers for Medical Applications, page 33-62. Woodhead Publishing, 2016.

[11] K. Miladi, D. Ibraheem, M. Iqbal, S. Sfar, H. Fessi, and A. Elaissari. Particles from preformed polymers as carriers for drug delivery. EXCLI J, $13: 28-57,2014$.

[12] K. Kogej. Thermodynamic analysis of the conformational transition in aqueous solutions of isotactic and atactic poly (methacrylic acid) and the hydrophobic effect. Polymers, 8(5) :168, 2016.

[13] S. Sugai and G. Ebert. Conformations of hydrophobic polyelectrolytes. Adv. Colloid Interface Sci, $24: 247-282,1985$.

[14] M. Wei, Y. Gao, X. Li, and M.J. Serpe. Stimuli-responsive polymers and their applications. Polym. Chem, 8(1) :127-143, 2017.

[15] C. Sester, F. Ofridam, N. Lebaz, E. Gagnière, D. Mangin, and A. Elaissari. ph-sensitive methacrylic acid-methyl methacrylate copolymer eudragit 1100 and dimethylaminoethyl methacrylate, butyl methacrylate, and methyl methacrylate tri-copolymer eudragit e100. Polym. Adv. Technol, 2019.

[16] A. Ghaffar, B. Yameen, M. Latif, and M.I. Malik. ph-sensitive drug delivery systems. In Metal Nanoparticles for Drug Delivery and Diagnostic Applications, page 259-278. Elsevier, 2020.

[17] F. Ofridam, N. Lebaz, É. Gagnière, D. Mangin, and A. Elaissari. Effect of secondary polymer on selfprecipitation of ph-sensitive polymethylmethacrylate derivatives eudragit e100 and eudragit 1100. Polym. Adv. Technol., Feb, 2020.

[18] K.T. Oh, T.K. Bronich, L. Bromberg, T.A. Hatton, and A.V. Kabanov. Block ionomer complexes as prospective nanocontainers for drug delivery. J. Controlled Release, 115(1) :9-17, 2006.

[19] E.D. Goddard. Polymer/surfactant interaction - its relevance to detergent systems. J. Am. Oil Chem. Soc, 71(1) :1-16, 1994.

[20] R. Bradbury, J. Penfold, R.K. Thomas, I.M. Tucker, J.T. Petkov, and C. Jones. Manipulating perfume delivery to the interface using polymer-surfactant interactions. J. Colloid Interface Sci, 466 :220-226, 2016.

[21] Y.V. Shulevich, T.H. Nguyen, D.S. Tutaev, A.V. Navrotskii, and I.A. Novakov. Purification of fatcontaining wastewater using polyelectrolyte-surfactant complexes. Sep. Purif. Technol, 113 :18-23, 2013.

[22] S.S. Ray, A.O.C. Iroegbu, and J.C. Bordado. Polymer-based membranes and composites for safe, potable, and usable water : A survey of recent advances. Chem. Afr, 2020.

[23] M.L. Sall. Toxicity and electrochemical detection of lead, cadmium and nitrite ions by organic conducting polymers : A review. Chem. Afr, 2020-07.

[24] B. Magny, I. Iliopoulos, R. Zana, and R. Audebert. Mixed micelles formed by cationic surfactants and anionic hydrophobically modified polyelectrolytes. Langmuir, 10(9) :3180-3187, 1994.

[25] P.S. Leung and E.D. Goddard. Gels from dilute polymer/surfactant solutions. Langmuir, 7(3) :608-609, 1991.

[26] P. Zhang, P.-Y. Zheng, F.-Y. Zhao, Q.-F. An, and C.-J. Gao. Preparation and pervaporation characteristics of novel ethanol permselective polyelectrolyte-surfactant complex membranes. RSC Adv, $5(78): 63545-63552,2015$.

[27] T. Nylander, Y. Samoshina, and B. Lindman. Formation of polyelectrolyte-surfactant complexes on surfaces. Adv. Colloid Interface Sci, 123-126:105-123, 2006.

[28] H.-W. Tseng, P.-C. Chen, H.-W. Tsui, C.-H. Wang, T.-Y. Hu, and L.-J. Chen. Effect of molecular weight of poly(acrylic acid) on the interaction of oppositely charged ionic surfactant-polyelectrolyte mixtures. J. Taiwan Inst. Chem. Eng, 92 :50-57, 2018.

[29] L. Chiappisi, I. Hoffmann, and M. Gradzielski. Complexes of oppositely charged polyelectrolytes and surfactants - recent developments in the field of biologically derived polyelectrolytes. Soft Matter, 9(15) :3896, 2013. 
[30] N. Khan and B. Brettmann. Intermolecular interactions in polyelectrolyte and surfactant complexes in solution. Polymers, 11(1) :51, 2018.

[31] E.D. Goddard and J.V. Gruber. Principles of Polymer Science and Technology in Cosmetics and Personal Care. CRC Press, 1999.

[32] E. Guzmán. Polymer-surfactant systems in bulk and at fluid interfaces. Adv. Colloid Interface Sci, $233: 38-64,2016$.

[33] D.Y. Chu and J.K. Thomas. Effect of cationic surfactants on the conformation transition of poly (methacrylic acid. J. Am. Chem. Soc, 108(20):6270-6276, 1986.

[34] K. Thalberg, B. Lindman, and K. Bergfeldt. Phase behavior of systems of polyacrylate and cationic surfactants. Langmuir, 7(12) :2893-2898, 1991.

[35] P.F.C. Lim, L.Y. Chee, S.B. Chen, and B.-H. Chen. Study of interaction between cetyltrimethylammonium bromide and poly(acrylic acid) by rheological measurements. J. Phys. Chem. B, 107(26) :6491-6496, 2003.

[36] N. Plucktaveesak, A.J. Konop, and R.H. Colby. Viscosity of polyelectrolyte solutions with oppositely charged surfactant. J. Phys. Chem. B, 107(32) :8166-8171, 2003.

[37] C. Wang and K.C. Tam. Interaction between polyelectrolyte and oppositely charged surfactant : Effect of charge density. J. Phys. Chem. B, 108(26) :8976-8982, 2004.

[38] Y. Li, S.M. Ghoreishi, J. Warr, D.M. Bloor, J.F. Holzwarth, and E. Wyn-Jones. Binding of sodium dodecyl sulfate to some polyethyleneimines and their ethoxylated derivatives at different ph values. Electromotive Force and Microcalorimetry Studies," Langmuir, 16(7) :3093-3100, 2000.

[39] D. Linke. Chapter 34 detergents. In Methods in Enzymology, volume 463, page 603-617. Elsevier, 2009.

[40] K. Pojják, E. Bertalanits, and R. Mészáros. Effect of salt on the equilibrium and nonequilibrium features of polyelectrolyte/surfactant association. Langmuir, 27(15) :9139-9147, 2011.

[41] A. Naderi, P.M. Claesson, M. Bergström, and A. Dėdinaitè. Trapped non-equilibrium states in aqueous solutions of oppositely charged polyelectrolytes and surfactants : effects of mixing protocol and salt concentration. Colloids Surf. Physicochem. Eng. Asp, 253(1-3) :83-93, 2005.

[42] E. Fegyver and R. Mészáros. The impact of nonionic surfactant additives on the nonequilibrium association between oppositely charged polyelectrolytes and ionic surfactants. Soft Matter, 10(12):1953-1962, 2014.

[43] R. Nagarajan. Thermodynamics of nonionic polymer-micelle association. Colloids Surf, 13 :1-17, 1985.

[44] P. Fisk. Chemical Risk Assessment: A Manual for REACH. John Wiley Sons, 2013.

[45] E. Guzmán. Effect of a natural amphoteric surfactant in the bulk and adsorption behavior of polyelectrolyte-surfactant mixtures. Colloids Surf. Physicochem. Eng. Asp, 585 :124178, 2020.

[46] O. Pyshkina, V. Sergeyev, A. Zezin, and V. Kabanov. Poly(n-ethyl-4-vinylpyridinium) cationzwitterionic surfactant complexes. Polym. Sci. Ser. B, 48 :271-273, 2006.

[47] A. Akanno, E. Guzmán, L. Fernández-Peña, F. Ortega, and R. G. Rubio. Surfactant-like behavior for the adsorption of mixtures of a polycation and two different zwitterionic surfactants at the water/vapor interface. Molecules, 24(19) :3442, 2019.

[48] A. Akanno, E. Guzmán, L. Fernández-Peña, S. Llamas, F. Ortega, and R. G. Rubio. Equilibration of a polycation-anionic surfactant mixture at the water/vapor interface. Langmuir, 34(25):7455-7464, 2018.

[49] S. Llamas. Towards understanding the behavior of polyelectrolyte-surfactant mixtures at the water/vapor interface closer to technologically-relevant conditions. Phys. Chem. Chem. Phys, $20(3)$ :1395-1407, 2018.

[50] A. Dedinaite and P.M. Claesson. Interfacial properties of aggregates formed by cationic polyelectrolyte and anionic surfactant. Langmuir, 16(4) :1951-1959, 2000.

[51] H. Wang, Y. Fan, and Y. Wang. Thermodynamic association behaviors of sodium dodecyl sulfate (sds) with poly(4-vinylpyridine n-oxide) (pvpno) at different ph values and ionic strengths. J. Surfactants Deterg, 20(3) :647-657, 2017. 